BMJ Open

Diabetes

Research

\& Care

\title{
Polyphenol-induced improvements in glucose metabolism are associated with bile acid signaling to intestinal farnesoid $X$ receptor
}

\author{
Kevin M Tveter, ${ }^{1}$ Jose A Villa-Rodriguez (D) , ${ }^{1}$ Alrick J Cabales, ${ }^{1}$ Li Zhang, ${ }^{2}$ \\ Fiona G Bawagan, ${ }^{1}$ Rocio M Duran, ${ }^{1}$ Diana E Roopchand (D) ${ }^{1}$
}

To cite: Tveter KM, Villa-Rodriguez JA, Cabales AJ, et al. Polyphenol-induced improvements in glucose metabolism are associated with bile acid signaling to intestinal farnesoid $X$ receptor. BMJ Open Diab Res Care 2020;8:e001386. doi:10.1136/ bmjdrc-2020-001386

\section{- Additional material is} published online only. To view please visit the journal online (http://dx.doi.org/10.1136/ bmjdrc-2020-001386).

KMT and JAV-R contributed equally.

Received 24 March 2020 Revised 2 June 2020 Accepted 22 June 2020

Check for updates

\section{(C) Author(s) (or their} employer(s)) 2020. Re-use permitted under CC BY-NC. No commercial re-use. See rights and permissions. Published by BMJ.

${ }^{1}$ Food Science, Rutgers, The State University of New Jersey, New Brunswick, New Jersey, USA

${ }^{2}$ Key Laboratory of Genomic and Precision Medicine, Beijing Institute of Genomics, Chinese Academy of Sciences, Beijing Branch, Beijing, China

\section{ABSTRACT}

Introduction Bile acid (BA) biotransformation by gut bacteria impacts BA profile and signaling to nuclear receptors, such as the farnesoid $X$ receptor (FXR) regulating glucose metabolism. Altered BA-FXR signaling was therefore investigated as a potential mechanism linking polyphenol-induced gut bacterial changes and improved glucose metabolism.

Research design and methods Diabetic $d b / d b$ were fed low-fat diet (LFD) or LFD supplemented with a proanthocyanidin-rich extract of grape polyphenols (LFDGP) for 4 weeks. Metabolic phenotypes, serum BAs, gut microbiota composition, and gene expression markers relevant to gut barrier and glucose metabolism were assessed. Gut organoids were used to investigate effects of individual BAs on ileal FXR activity.

Results Compared with LFD-fed controls, GP supplemented $d b / d b$ mice showed improved glucose metabolism, decreased relative abundance of gut bacteria associated with production of secondary BAs (SBAs), and depleted serum levels of SBAs taurohyodeoxycholic acid (THDCA), $\omega$-muricholic acid ( $\omega M \mathrm{MCA})$, and tauro-

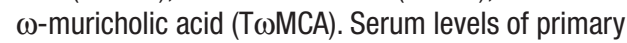
BAs (PBAs) increased, consistent with higher gene expression of PBA synthesis enzyme Cyp7a1. GP-induced BA changes associated with FXR inhibition as evidenced by reduced expression of FXR-responsive genes Shp, Fgf15, and Fabp6 in ileum tissue as well as hepatic Shp, which negatively regulates PBA synthesis. GP treatment did not affect expression of hepatic Fxr or expression of $A b c b 11$, Slc51b, and Obp2a genes controlling BA transport. Ceramide biosynthesis genes Smpd3, Spt/c2, and Cers4 were decreased in liver and intestine suggesting lower tissue ceramides levels may contribute to improved glucose metabolism. THDCA, $\omega$ MCA, and T $\omega$ MCA behaved as FXR agonists in ileal organoid experiments; therefore, their depletion in serum of GP-supplemented $d b / d b$ and wild type (WT) mice was consistent with FXR inhibition. Conclusion These data suggest that by altering the gut microbiota, GPs modify BA-FXR signaling pathways to promote glucoregulation.

\section{INTRODUCTION}

Dietary polyphenols in plant-based foods contribute to improved glycemic control in

\section{Significance of this study}

What is already known about this subject?

- Dietary polyphenols, such as proanthocyanidins, alter the gut microbial community and are associated with improved metabolic resilience in humans and mice.

- Bile acids (BAs) signal to farnesoid X receptor (FXR), a nuclear transcription factor that regulates hepatic BA synthesis and glucose metabolism.

What are the new findings?

- Grape polyphenol (GP) supplementation decreased abundances of gut bacterial taxa associated with secondary BA (SBA) production concomitant with depletion of SBAs and increased primary BAs (PBAs) in murine serum.

- GP supplementation suppressed expression of FXRregulated genes Fgf15, Fabp6, and Shp, an inhibitor of PBA synthesis, which was consistent with increased serum PBAs.

- GP-induced FXR inhibition was associated with decreased expression of genes required for biosynthesis of ceramides, which impair glucose homeostasis.

- The SBAs depleted in GP-treated mice were revealed as FXR agonists in ileal gut organoids.

How might these results change the focus of research or clinical practice?

- This study highlights BA-FXR signaling pathways as an important mechanism for further investigation in human intervention studies of dietary polyphenols and metabolic health.

mice and humans. ${ }^{1-3}$ Improved glucose metabolism in mice supplemented with berry/ fruit extracts was related to a proanthocyanidin (PAC)-induced bloom of Akkermansia muciniphila, ${ }^{124}$ a microbe shown to attenuate symptoms of metabolic syndrome (MetS) and type-2 diabetes (T2D) in obese mice and humans. ${ }^{5}$ We hypothesized that metabolic improvements also result from changes in 
host-derived bacterial metabolites regulating host energy metabolism.

Bile acids (BAs) are signaling molecules linking the gut microbiota to host energy metabolism. Primary BAs (PBAs) synthesized in the liver are conjugated with taurine (mice) or glycine (humans). ${ }^{7}$ Bacterial-derived bile salt hydrolases deconjugate taurine or glycine from the sterol core of PBAs followed by bacterial transformations, such as 7 $\alpha / \beta$-dehydroxylation, dehydrogenation, and epimerization, which generate secondary BAs (SBAs). ${ }^{7}$ PBAs and SBAs signal to key regulators of energy metabolism, such as nuclear transcription factor farnesoid $\mathrm{X}$ receptor (FXR) and Takeda G protein-coupled receptor 5 (TGR5) ${ }^{7} \mathrm{Fxr}^{-1-}$ mice were protected from high-fat diet (HFD)-induced obesity and had altered BA and gut microbiota (higher Bacteroidetes, less Firmicutes) profiles compared with wild type (WT) mice. ${ }^{8}$ Transfer of cecal microbiota from HFD-fed $\mathrm{Fxr}^{-1-}$ mice to germ free WT mice resulted in less adiposity and improved glucose metabolism compared with mice that received microbiota from HFD-fed WT mice, suggesting the altered gut microbial and BA profiles in FXR deficient mice contributed to metabolic improvements. ${ }^{8}$ Studies using tissue-specific FXR knockout mice showed that intestinal FXR activity was required to mediate HFD-induced metabolic dysfunctions. ${ }^{9} 10$ Pharmacological inhibition of intestinal FXR using tempol, antibiotics, metformin, or glycine- $\beta$-muricholic acid (Gly-MCA) led to gut microbial remodeling and improved glucose and lipid homeostasis. ${ }^{9-12}$

In addition to the A. muciniphila bloom, mice fed HFDsupplemented with PAC-rich grape polyphenols (GPs) showed other profound bacterial community changes raising the possibility that altered $\mathrm{BA}$ profile and signaling could contribute to observed improvement in glucose homeostasis. ${ }^{14}$ The present study provides compelling evidence in support of this hypothesis.

\section{RESEARCH DESIGN AND METHODS}

Diets

A complex of $10 \%$ grape polyphenols and soy protein isolate (GP-SPI) was prepared as previously described. ${ }^{1413}$ Nutritional profiles for SPI and GP-SPI are provided in online supplementary table 1 . Isocaloric, ingredient-matched diets (Research Diets, New Brunswick, New Jersey, USA) used in this study were previously described. ${ }^{4}$ Mice were fed lowfat diet (LFD) containing 10\% SPI, LFD formulated with 10\% GP-SPI delivering 1\% GP (LFD-GP), HFD containing $10 \%$ SPI or HFD formulated with $10 \%$ GP-SPI delivering $1 \%$ GP (HFD-GP). Online supplementary table 2 provides diet formulation details.

\section{Animals}

Four-week-old $d b / d b$ mice (B6.BKS(D)-Lepr $r^{d b} / \mathrm{J}$; stock no. 000697; Jackson Laboratory, Bar Harbor, Maine, USA) were single-housed on a 12-hour light/dark cycle (07:00 to 19:00 hours light) with ad libitum access to LFD and water in a controlled-temperature room $\left(24^{\circ} \mathrm{C} \pm 1^{\circ} \mathrm{C}\right)$ for 1 week for acclimation. Mice were randomized to receive LFD $(\mathrm{n}=7)$ or LFD-GP $(\mathrm{n}=7)$ for 28 days. Metabolic phenotyping included food intake, body weight, body composition (EchoMRI 3-in-1 system; Echo Medical Systems, Houston, Texas, USA), and oral glucose tolerance tests, as previously described. ${ }^{14}$ On day 29, mice were euthanized by $\mathrm{CO}_{2}$ inhalation, followed by cardiac puncture and collection of tissues, as previously described. ${ }^{14}$ Fecal and cecal samples were collected for microbial community profiling. Wild type C57BL/6J mice $(\mathrm{n}=10)$ aged 5 weeks were acclimated on LFD for 1 week and randomly divided into two groups and fed HFD or HFD-GP ( $\mathrm{n}=5$ per group) for 10 weeks after which mice were euthanized by $\mathrm{CO}_{2}$ inhalation.

\section{Tissue gene expression analysis}

RNA was extracted from ileum, jejunum, colon, and liver (30-60 mg) with RNeasy Plus Universal Mini Kit (QIAGEN) followed by RNA cleanup (Machery-Nagel, RNA purification kit). RNA ( $5 \mu \mathrm{g})$ was reverse transcribed to cDNA and qPCR was performed using TaqMan primers (online supplementary table 4) as previously described. ${ }^{4}$ Data were analyzed using $2^{-\Delta \mathrm{CT}}$ method using hydroxymethylbilane synthase (HMBS) as housekeeping gene.

qPCR of A. muciniphila in fecal and cecal samples was performed as previously described. ${ }^{1}{ }^{4}$ Briefly, gDNA extracted from fecal/cecal samples was diluted to $2.5 \mathrm{ng} /$ $\mu \mathrm{L}$ for quantification of A. muciniphila abundance relative to total bacteria and archaea by qPCR using A. muciniphila (AM1, AM2) and universal primer (U341F, U515R) sets. ${ }^{1}$

\section{S rRNA gene sequencing}

Genomic DNA was extracted from 84 fecal and 14 cecal samples collected from $d b / d b$ mice. Illumina protocols were used to prepare V4 amplicons of the 16S rRNA gene for sequencing on a MiSeq system, resulting in $>1.099 \times 10^{7}$ reads. Denoising and clustering were conducted using DADA2 algorithm to differentiate sequences into amplicon sequence variants (ASVs) for downstream analysis using QIIME 2.0. ${ }^{15}$ Details are available in online supplementary materials.

\section{Serum biochemistry}

Serum for BA analysis was collected by cardiac puncture. BAs were analyzed on a Water's Alliance e2695 HPLC system (Waters Milford, Massachusetts, USA) coupled to a Water's Acquity QDA mass spectrometer equipped with an electrospray interphase (ESI; Waters Milford, Massachusetts, USA) and quantified by external calibration curves using $>95 \%$ pure standards. Details are provided in online supplementary materials. Serum leptin, polypeptide YY (PYY), interleukin-6 (IL-6) and insulin were determined using a MILLIPLEX MAP Mouse Metabolic Hormone Magnetic Bead kit (Millipore) with a MagPix instrument (Luminex), as previously described. ${ }^{4}$

\section{Organoid experiments}

Intestinal crypts were isolated from WT C57BL/6J mouse ileum, according to established methods. ${ }^{16}$ Crypts were counted and added to Matrigel (five crypts per $\mu \mathrm{L}$ ) 
(Corning 356231, growth factor reduced) and $25 \mu \mathrm{L}$ was added per well (in 48-well plates) and allowed to polymerize $\left(37^{\circ} \mathrm{C}\right.$ for $\left.15-20 \mathrm{~min}\right)$, followed by addition of $1 \mathrm{~mL}$ of complete growth medium (CGM, see online supplementary materials). CGM (without Y-27632 dihydrochloride monohydrate) was replaced every $2-3$ days. Organoids were passaged every 5-7 days (1:3 ratio). Mature organoids (4 days postpassage) were treated in triplicate $(n=3$ wells) with $5 \%$ methanol (vehicle), chenodeoxycholic acid (CDCA) $(100 \mu \mathrm{M})$ alone, or CDCA plus another BA (200 $\mu \mathrm{M})$ in CGM for 17 hours. RNA was extracted for qPCR analysis using TaqMan primers, as described above. ${ }^{4}$

\section{Statistical analyses}

Analyses were conducted using Prism 8.0.2 (GraphPad Software, La Jolla, California, USA). Significant differences (two groups) were assessed with a two-tailed, unpaired Student's $t$ test with Welch correction for unequal variance when needed or by one-way or twoway analysis of variance ( $>2$ groups) followed by Sidak's or Tukey's multiple comparison test. Statistical analysis of alpha and beta-diversity metrics was calculated using QIIME 2 (details in online supplementary materials). ADONIS and permutation analysis were conducted using R Studio V.3.4.2 (R Studio Software, Boston, Massachusetts, USA) and Python 3.7.1.

\section{RESULTS}

GPs improve glucose metabolism in $d b / d b$ mice independent of obesity

Leptin receptor-deficient $d b / d b$ mice develop obesity, gut barrier dysfunction, and hyperglycemia independent of HFD feeding. ${ }^{17}$ Compared with LFD-fed controls, $d b / d b$ mice fed LFD containing PAC-rich GPs (LFD-GP) for 4 weeks showed significantly improved oral glucose tolerance (figure 1A). ${ }^{4}$ Area under the curve remained stable over time for the LFD-GP group but increased in the LFD group (figure 1A). Mice fed LFD-GP initially exhibited a transient decrease in food intake presumably due to taste, but at later time points LFD and LFD-GP groups consumed similar amounts of food $(4.99 \pm 0.69$ and $4.29 \pm 0.69 \mathrm{~g} /$ day/mouse, respectively, $\mathrm{p}>0.05$, online supplementary figure $1 \mathrm{~A})$. The LFD-GP group consumed $42.9 \pm 6.9 \mathrm{mg}$ of GPs per day. Both groups had similar body weight gain, body composition, and liver weights (online supplementary figure 1B-D).

\section{GPs promote a bloom in A. muciniphila without improving markers of metabolic endotoxemia}

Compared with controls, GP-supplemented $d b / d b$ mice had decreased $\alpha$-diversity as evidenced by richness, Shannon index, and Faith's phylogenetic diversity index (figure 2A). Principal coordinate analysis showed that GPs significantly altered fecal community structure within 2 days (online supplementary figure 2). As previously observed, ${ }^{14}$ GPs promoted increased cecal mass (online supplementary figure
1E), a phenotype common to antibiotic-treated and germ-free mice and consistent with reported antibacterial properties of PACs. ${ }^{18} 19$ GP-supplemented mice had an increased relative abundance of phylum Verrucomicrobia at the expense of Firmicutes in fecal (days 6-27) and ceca samples (day 27) (figure 2B,C, online supplementary figure 3A,B). Ceca of GP-supplemented mice had higher relative abundance of Bacteroidetes (25\% >LFD) although, except for day 13, fecal Bacteroidetes remained similar between groups (figure 2B, online supplementary figure 3C). GP supplementation did not consistently alter levels of Proteobacteria or Actinobacteria (figure 2B, online supplementary figure 3D,E). Consistent with increased Verrucomicrobia, quantitative qPCR analyses confirmed that GPs promoted a bloom in Akkermansia muciniphila (figure 2C) at the expense of other taxa (figure 2D).

Reduced abundance of $A$. muciniphila and metabolic endotoxemia (characterized by gut dysbiosis, compromised gut barrier integrity, lipopolysaccharide (LPS) leakage, and intestinal inflammation) was associated with impaired glucose metabolism in obese mice and humans. ${ }^{5620}$ Oral administration of A. muciniphila in obese, diabetic mice and humans resulted in improved glucose homoeostasis and attenuated metabolic endotoxemia through improved gut barrier integrity. ${ }^{5620}$ GP-induced improvement in glucose metabolism in HFD-fed mice has therefore been considered a consequence of the A. muciniphila bloom leading to reduced inflammation and increased gut barrier integrity. ${ }^{124}$ Reduced metabolic endotoxemia could not, however, explain the improved glucose tolerance in GP-supplemented $d b / d b$ mice despite increased A. muciniphila $(\sim 15 \%)$ in feces (day 27 ) and cecum (figure 2C). Relative to control, GP-supplementation did not change intestinal gene expression of markers of inflammation ( Tnf, Il-6, iNOS), gut barrier integrity (Tjp1, Ocln, Muc2), peripheral lipid deposition (Fiaf) or glucose transport (Glut2) (online supplementary figure $4 \mathrm{~A}-\mathrm{C})$. GP-supplemented $d b / d b$ mice had less $M u c 3$ expression in jejunum and ileum (online supplementary figure $4 \mathrm{~A}, \mathrm{~B}$ ), suggesting lower mucus secretion. There were no differences in serum insulin, IL-6, or glucoregulatory hormones PYY and leptin (online supplementary table 3 ). These data suggested that other mechanism(s) were driving improved glucose metabolism.

\section{GPs reduced gut bacterial taxa associated with production of} SBAs

Although total bacterial and archaeal abundance was not significantly different between LFD and LFD-GP groups (figure 2E), GPs induced profound gut microbial changes (online supplementary figure 5) that would be expected to alter BA diversity, abundance, and signaling. Targeted liquid chromatography-mass spectrometry (LC-MS) analysis revealed that GP-supplemented $d b / d b$ mice had higher serum concentrations of 
$\square$ LFD $\square$ LFD-GP
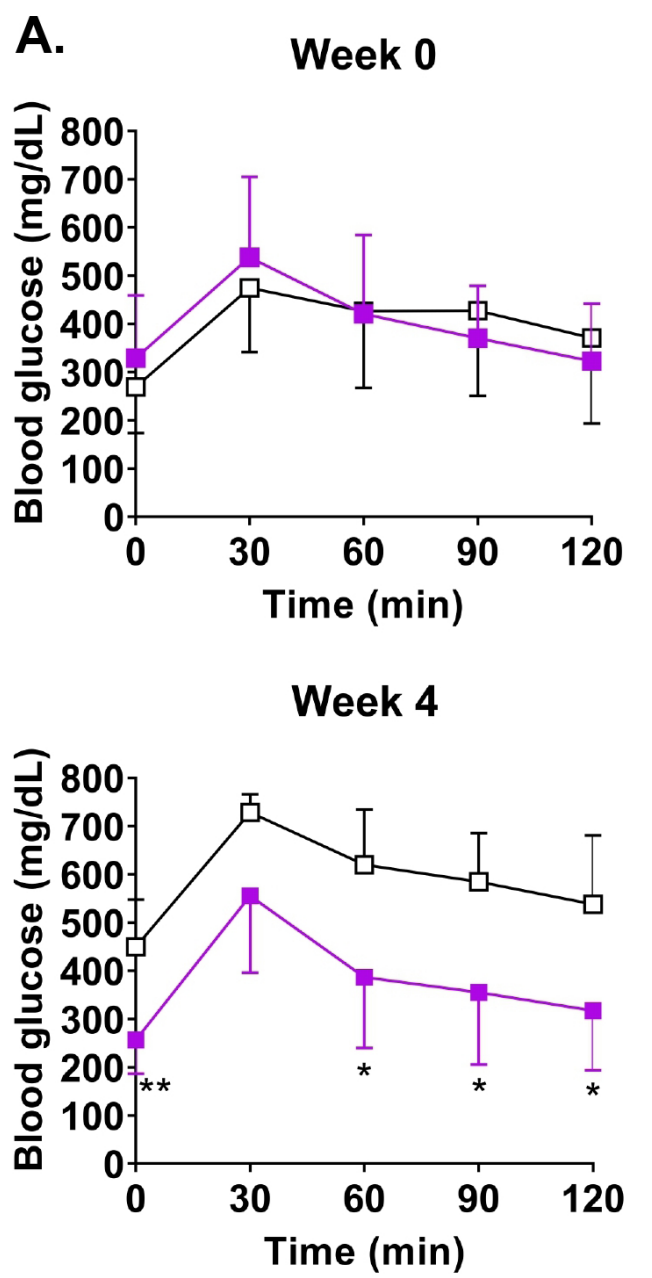

Week 2

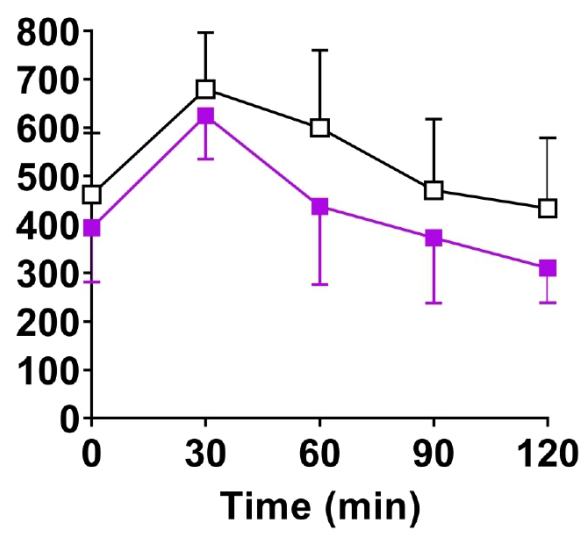

B.

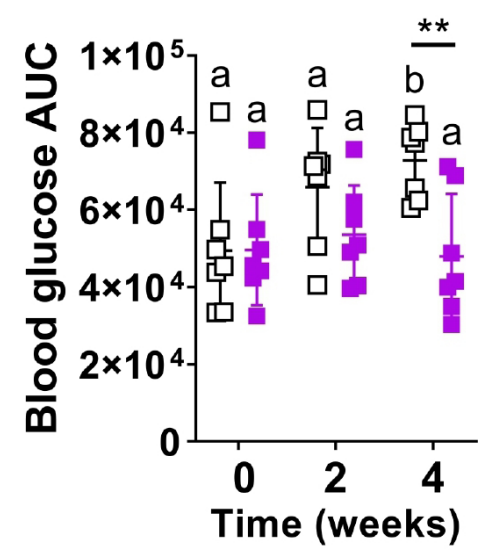

Figure 1 GPs improve glycemic control in obese $d b / d b$ mice. (A) Oral glucose tolerance tests were performed after mice consumed LFD (open squares; $n=7$ ) or LFD-GP (closed squares; $n=7$ ) for 0,2 , and 4 weeks. Blood glucose concentrations $(\mathrm{mg} / \mathrm{dL})$, expressed as mean $\pm \mathrm{SD}$, were measured at the indicated time points $(0-120 \mathrm{~min})$ following administration of glucose $(2 \mathrm{~g} / \mathrm{kg})$. Between group difference was determined by unpaired, two-tailed $t$-test with Welch's correction $\left({ }^{*} \mathrm{p}<0.1,{ }^{* *} \mathrm{p}<0.01\right)$. (B) Scatter plot of blood glucose AUC determined for individual mice at weeks 0,2 and 4, where mean AUC \pm SD are shown as horizontal and vertical bars. Difference was determined using two-way ANOVA followed by Tukey's (intragroup) posthoc test (different letters indicate significant difference, $p<0.05$ ) or by Sidak's (intergroup) posthoc test $\left({ }^{\star \star} p<0.01\right)$. ANOVA, analysis of variance; AUC, area under the curve; GP, grape polyphenol; LFD, low-fat diet.

PBAs driven by increased cholic acid (CA) and taurocholic acid (TCA) (figure $3 \mathrm{~A}$ and $\mathrm{C}$, online supplementary figure $6 \mathrm{~A})$. Concentrations of $\beta$-muricholic acid ( $\beta$ MCA), tauro- $\beta$ MCA (T $\beta$ MCA), tauro- $\alpha$ MCA (T $\alpha \mathrm{MCA})$, taurochenodeoxycholic acid (TCDCA) were similar between groups; $\alpha$ MCA was not detected in LFD-GP group (figure 3A, online supplementary figure $6 A)$. Increased PBA pool correlated with an overall reduction in SBAs (figure 3C) where $\omega \mathrm{MCA}$, T $\omega \mathrm{MCA}$, and taurohyodeoxycholic acid (THDCA) were undetectable in $d b / d b$ mice fed LFD-GP, although deoxycholic acid (DCA) was increased (figure 3A). Total serum BAs were similar between groups (figure 3B).

The GP-induced depletion of SBAs was unrelated to the leptin receptor mutation in $d b / d b$ mice. Compared with HFD-fed controls, wild type C57BL6/J mice fed HFD supplemented with $1 \%$ GP (HFD-GP) for 10 weeks also showed serum depletion of SBAs THDCA, $\omega$ MCA, and T $\omega \mathrm{MCA}$, reduced overall levels of serum SBAs, and no difference in concentration of total serum BAs (online supplementary figures $6 \mathrm{~B}$ and 7). Unlike $d b / d b$ mice, GP-supplemented WT mice had decreased taurodeoxycholic acid (TDCA) and no significant difference in CA or overall PBA pool, although TCA concentration was increased (online supplementary figures $6 \mathrm{~B}$ and 7 ).

In $d b / d b$ mice, multiple correlation analyses were performed to associate GP-induced changes in serum BAs (figure 3) to changes in fecal/cecal gut bacteria (online supplementary figure 5). The GP-induced increase of CA, TCA (PBAs), and DCA (SBA) was positively and significantly associated with increased abundance of Akkermansia, Blautia, Clostridium (ASV-59), and S24-7 (figure 3E,F). Blautia and Clostridium possess 

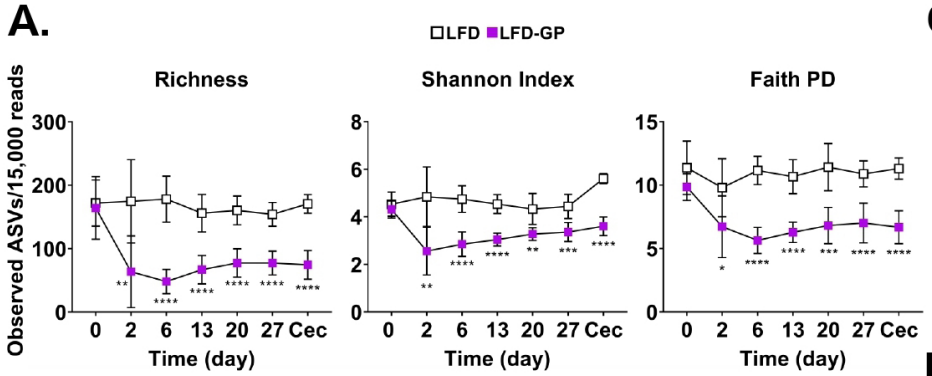

B. 100

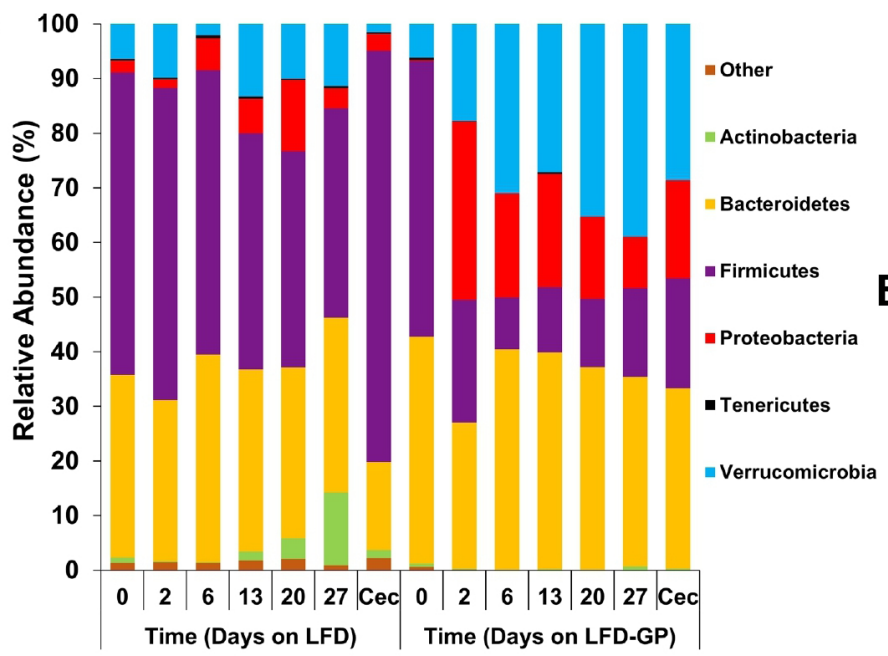

C.

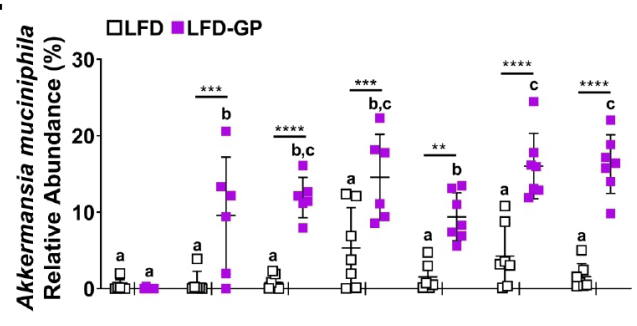

Figure 2 Supplementation of GPs remodeled gut microbial composition and diversity. (A) Microbial $\alpha$-diversity metrics of fecal samples collected from mice after consuming LFD (open squares; $n=7$ ) or LFD-GP (closed squares; $n=7$ ) for indicated number of days (0-27) and of cecal (Cec) samples collected at endpoint (day 29). Difference was determined by two-way ANOVA followed by Sidak's test. (B) Per cent relative abundance of main bacterial phyla based on Naïve-Bayes taxonomic classifier (non-rarified data). Phyla present at $<0.3 \%$ relative abundance were classified as 'Other'. Percentage of (C) $A$. muciniphila DNA and (D) non-A. muciniphila DNA relative to total bacterial and archaeal DNA in fecal and cecal samples, where relative abundance was determined by qPCR using primers specific for $A$. muciniphila (AM1/AM2) and universal V4 primers (515F/806R). (E) Total 16S bacterial and archaeal gene counts/ng of gDNA extracted from fecal or cecal samples. Group mean $\pm S D$ at each time point are illustrated by horizontal and vertical lines. Difference between diet groups over time (panels C-E) was determined using two-way ANOVA followed by Sidak's posthoc test (intergroup comparison) or Tukey's posthoc test (intragroup comparison). Different letters $(a, b$ and $c)$ indicate significant difference within diet groups $(p<0.05)$, while the same letter indicates no difference. Between group differences (panels A, C, D, E): ${ }^{\star} p<0.05,{ }^{* \star} p<0.01,{ }^{* * *} p<0.001,{ }^{* * *} p<0.0001$. ANOVA, analysis of variance; ASV, amplicon sequence variants; AUC, area under the curve; GP, grape polyphenol; LFD, low-fat diet; PD, phylogenetic diversity.

$7 \alpha$-dehydroxylating activity; therefore, taxa within these genera may be responsible for DCA production via dehydroxylation of CA. ${ }^{21}$ GP-treated mice had lower levels of taxa within the Clostridiales order (ASV-50 and 51), Ruminococcaceae and Lachnospiraceae families (ie, ASV-56, 57, 61, 65, 70, 75, and 76), and Clostridium genus (ASV-74) (online supplementary figure 5 ), which are reported to possess $7 \alpha$-dehydroxylating activity required for conversion of PBAs to SBAs. ${ }^{21-24}$ In agreement with evidence from mice and humans, the GP-induced decrease in these bacterial taxa encoding $7 \alpha$-dehydroxylation activity was highly correlated to the

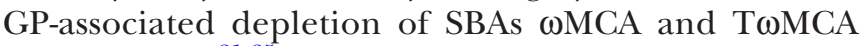
(figure $3 \mathrm{E}, \mathrm{F}) .^{21}{ }^{25}$ Finally, positive and significant associations were found between reduced $\alpha \mathrm{MCA}, \mathrm{T} \omega \mathrm{MCA}$, and/or $\omega \mathrm{MCA}$ and reductions in taxa belonging to RF39, Anaeroplasma, Ruminococcus, Butyricicoccus, Dorea,
Dehalobacterium, Christensenellaceae, Lactococcus, Streptococcus, and Oscillospira (figure 3E,F).

\section{GP-associated BA changes promote inhibition of FXR}

signaling and upregulation of classical BA synthesis pathway To investigate the consequences of GP-induced serum BA changes, gene expression of FXR, TGR5 and their downstream targets were analyzed in tissues. GP supplementation did not change ileal Fxr gene expression; however, FXR transcriptional activity was decreased as expression of its target genes, fibroblast growth factor 15 (Fgf15), small heterodimer partner $(S h p)$, and ileal BA binding protein (I-BABP, gene Fabpo) were suppressed in ileum (figure 4A). Intestinal FXR signaling negatively regulates hepatic PBA synthesis through interaction of Fgf15 with hepatic fibroblast growth factor receptor $4 / \beta$ Klotho receptor complex or by regulating 
A. $\quad$ LFD $\triangle$ LFD-GP

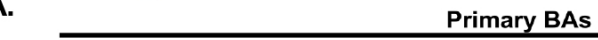

$d b / d b$ mice (B6.BKS(D)-Lepr $\left.r^{d b} / J\right)$

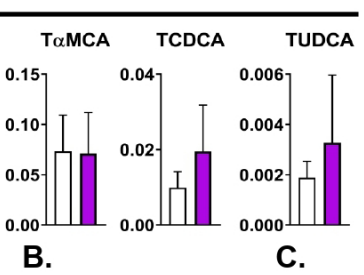

D.

$\square$ Primary BAs

- Secondary BAs
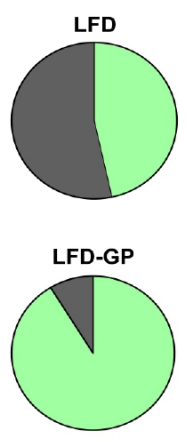

E.

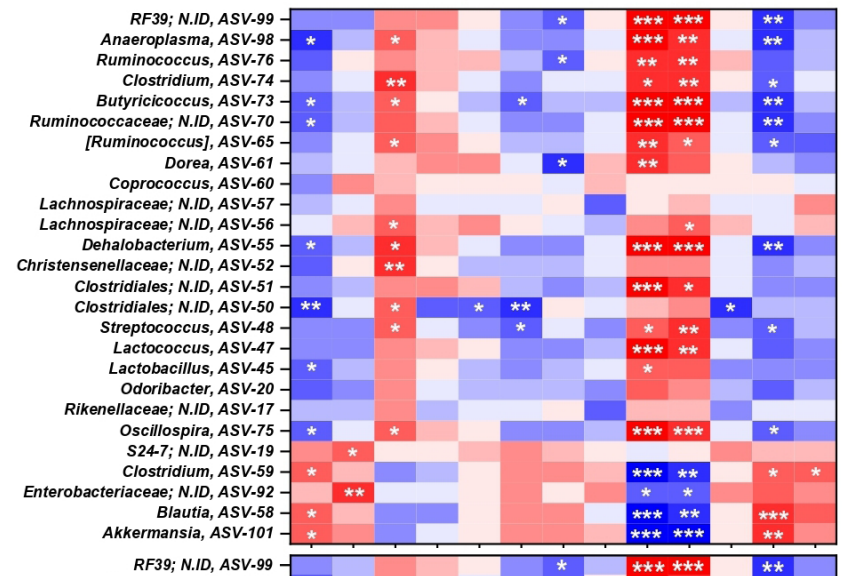

F.
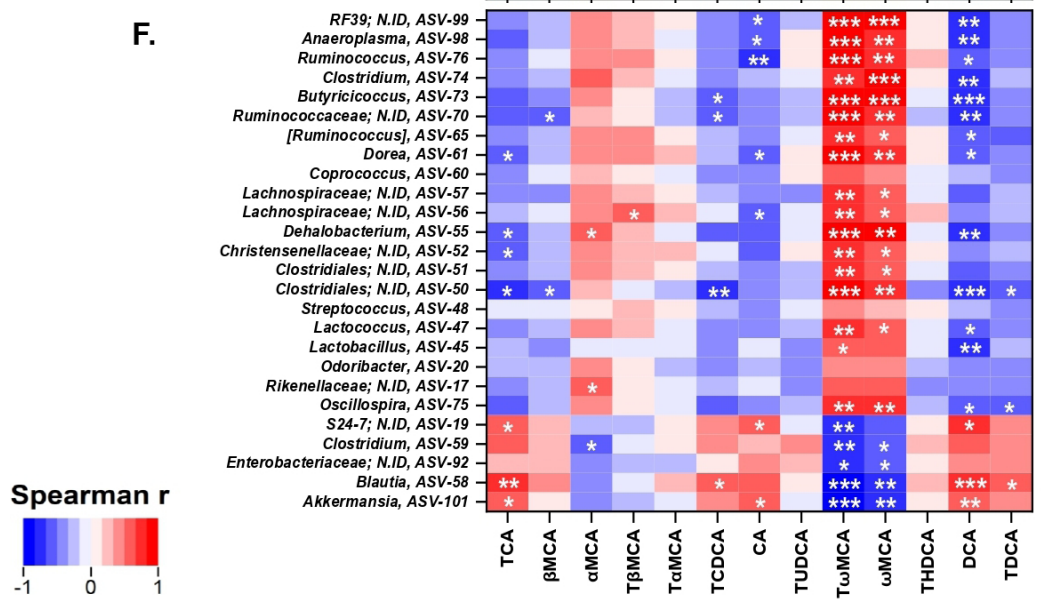

Figure 3 GPs increased PBAs and reduced SBAs in serum in association with depletion of bacterial ASVs related to SBA production. (A) LC-MS and pure standards were used to determine the mean concentration of individual PBAs and SBAs (mean $\pm S D$ ) in serum samples ( $n=7$ samples) collected from individual mice fed LFD (white bars) or LFD-GP (purple bars). (B) Total serum BA concentration (mean \pm SD) was determined based on sum of individual PBA and SBA concentrations (shown in panel A) quantified for each mouse fed LFD $(n=7)$ or LFD-GP $(n=7)$. (C) Serum PBA and SBA concentrations (mean \pm SD) in LFD versus LFD-GP diet groups were calculated by summing the individual PBAs or SBAs shown in panel A. (D) Using data from panels B and C, pie charts illustrate pooled PBAs (green) and pooled SBA (gray) as a percentage of total serum BA concentration quantified for LFD and LFD-GP groups. For panels A-C, significant difference was determined using unpaired, two-tailed $t$-test followed by Welch's correction: ${ }^{*} p<0.05$, ${ }^{\star *} p<0.01$. Heatmap representation of the Spearman's $r$ correlation coefficient between serum BA profile and significantly changed bacterial ASVs (at genera or family level of taxonomy) in GP-treated mice (relative to control diet group) from (E) day-27 fecal samples or (F) day-29 cecal samples. Shades of red indicate serum BA and bacterial taxa are positively correlated $(0$ to +1$)$ while shades of blue indicate a negative correlation $(0$ to -1$)$. Significant positive or negative correlations are shown: ${ }^{*} p<0.05,{ }^{* *} p<0.01,{ }^{* * *} p<0.001$. ASV, amplicon sequence variant; CA, cholic acid; DCA, deoxycholic acid; GP, grape polyphenol; LC-MS, liquid chromatography-mass spectrometry; LFD, low-fat diet; MCA, muricholic acid; PBA, primary bile acid; SBA, secondary bile acid; TCA, taurocholic acid; TCDCA, taurochenodeoxycholic acid; TDCA, tauro-deoxycholic acid; THCDA, taurohyodeoxycholic acid; T $\alpha$ MCA, tauro- $\alpha-M C A$; T $\beta$ MCA, tauro- $\beta-M C A ;$ T $\omega$ MCA, tauro- $\omega-\mathrm{MCA}$; TUDCA, tauro-ursodeoxycholic acid. 
$\square$ LFD $\square$ LFD-GP

A. lleum

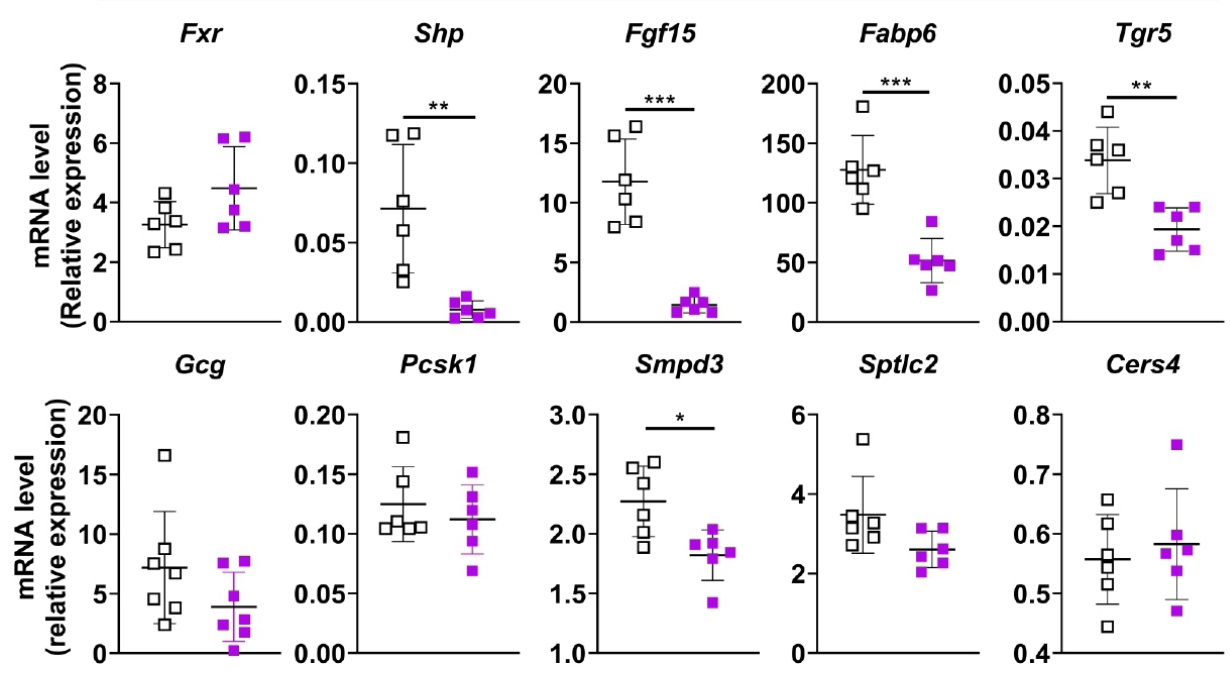

B. Liver

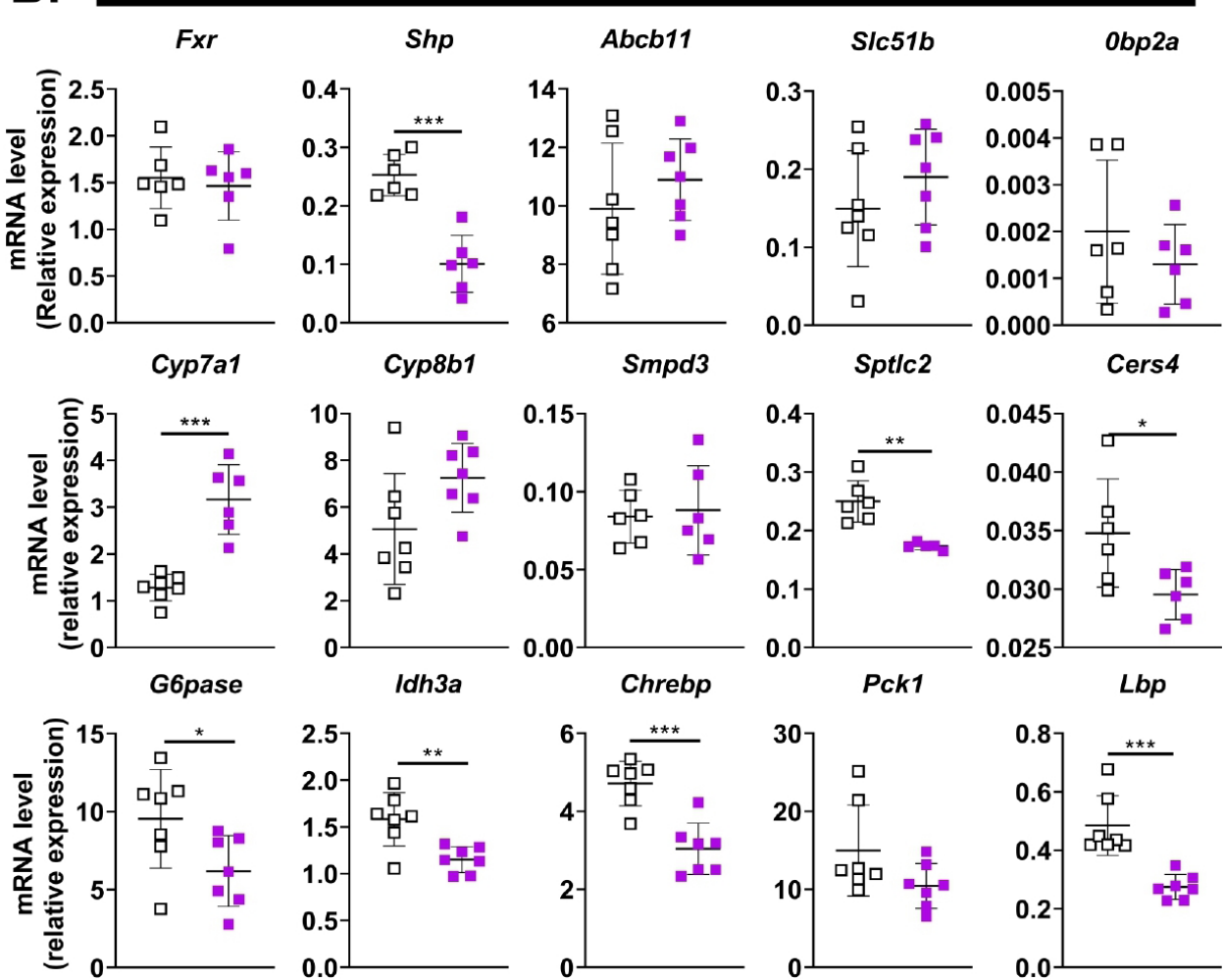

Figure 4 Expression of genes involved in FXR signaling, ceramide synthesis, and glucose metabolism in response to GPsupplementation. Scatter plot of relative mRNA levels of indicated genes expressed in (A) ileum and (B) liver tissues collected from individual mice fed LFD (open squares) or LFD-GP (closed squares). Group mean $\pm S D$ ( $n=7$ samples /group) is illustrated by horizontal and vertical lines. Data represent qPCR of technical duplicates analyzed by $2^{-\triangle C T}$ method. Between group difference was determined by unpaired, two-tailed $t$-test with or without Welch's correction for unequal variance: * $p<0.05$, ${ }^{* \star} \mathrm{p}<0.01,{ }^{\star \star \star} \mathrm{p}<0.001$. FXR, farnesoid X receptor; GP, grape polyphenol; LFD, low-fat diet.

the hepatic expression and gene-repressive function of Shp via liver receptor homologue-1 and hepatocyte nuclear factor- $4 \alpha .^{26-29}$

In agreement with reduced ileal $\mathrm{Fg} f 15$, hepatic $S h p$ expression was significantly decreased (figure 4B). Fgf15 and Shp negatively regulate BA synthesis; therefore, their reduced expression was consistent with hepatic upregulation of cytochrome P450 family 7 subfamily A member 1 (Cyp7a1), the rate-limiting enzyme in the classical BA synthesis pathway, ${ }^{7}$ and a trending increase in downstream enzyme Cyp8b1 ( $\mathrm{p}=0.06$; figure 4B). Gene expressions of Cyp27a1 and Cyp7b1 (online 
supplementary figure $4 \mathrm{E}$ ), involved in the alternative BA synthesis pathway, were unaffected by GP treatment. To determine if reduced hepatic Shp expression altered hepatic FXR activity, we examined FXR target genes Abcb11 encoding bile salt export pump (Bsep), Slc51b encoding organic solute transporter- $\beta$ (Ost- $\beta$ ), ${ }^{30}{ }^{31}$ and Obp2a encoding lipocalin 13 (Lpn13), an acute phase protein. ${ }^{32}$ Compared with control, GP treatment did not affect hepatic expression of Fxr, Abcb11, Slc51b, or Obp2a (figure 4B). These data suggest that GP-mediated effects on FXR activity are restricted to intestine resulting in suppression of ileal $F g f 15$ and $S h p$ transcription and increased Cyp7a1 activity and PBA synthesis (figure 3).

FXR activity regulates $\operatorname{Tgr} 5$ expression; therefore, we measured Tgr 5 mRNA levels and downstream targets. ${ }^{33}$ On activation by BAs, TGR5 signals the release of incretin glucagon-like peptide-1 (Glp-1) to promote euglycemia. ${ }^{33}$ Glp-1 is produced when proglucagon protein $(G c g)$ is cleaved by prohormone convertase $1 / 3$ (PC1/3), encoded by Pcsk $1 .{ }^{34}$ GP-supplemented mice showed reduced ileal expression of $\operatorname{Tgr} 5$ and no changes in Gcg or Pcsk1 (figure 4A). GPs did not change expression of $F x r, F g f 15$, or $T g r 5$ in colon tissue. GPs induced an increase in colonic $G c g$, however, Pcsk1 was unchanged indicating Glp-1 levels were unaffected (online supplementary figure 4D). These data further support the idea that GPs inhibit ileal FXR.

\section{GP-mediated FXR inhibition is associated with \\ downregulation of ceramide synthesis genes and improved markers of hepatic energy metabolism}

Tissue accumulation of ceramides is linked to insulin resistance and diabetes, which can be ameliorated by pharmacological or genetic inhibition of ceramide biosynthesis. ${ }^{35}$ FXR activity positively upregulates genes required for ceramide synthesis in ileum, which leads to impaired glucose metabolism and hepatic steatosis in mouse models of MetS/T2D. ${ }^{10}{ }^{36}$ Synthesis and accumulation of ceramides in liver contributes to hepatic insulin resistance, steatohepatitis, and metabolic disease. ${ }^{35}{ }^{37}$ We found that GP-induced FXR inhibition was associated with lower expression of de novo ceramide synthesis genes, specifically sphingomyelin phosphodiesterase 3 (Smpd3) in ileum (figure 4A) and serine palmitoyltransferase long-chain base subunit 2 (Sptlc2) and ceramide synthase 4 (Cers4) in liver (figure 4B). Consistent with lower expression of ceramide biosynthesis genes, GP-supplemented mice showed improvements in markers of hepatic energy metabolism, evidenced by lower hepatic expression of: (1) carbohydrate response element binding protein (Chrebp), a transcription factor that activates key enzymes of de novo lipogenesis; ${ }^{38}$ (2) glucose6 -phosphatase (G6Pase), which catalyzes the final step in hepatic glucose production; and (3) Idh $3 a$, a subunit of the IDH3 isocitrate dehydrogenase heterotetramer complex that regulates fatty acid metabolism and whose inhibition is associated with hepatic glycogen synthesis (figure 4B). ${ }^{39}$ GP-supplemented mice had reduced expression of $L b p$, encoding LPS-binding protein, suggesting decreased liver inflammation and insulin resistance. ${ }^{40}$ Hepatic gene expression of gluconeogenesis enzyme phosphoenolpyruvate carboxykinase $(P c k 1)$ and $\mathrm{C} / \mathrm{EBP}$ homologous protein $(C h o p)$, which are normally upregulated during hepatic endoplasmic reticulum stress, ${ }^{41}$ were similar between groups (online supplementary figure $4 \mathrm{E}$ ).

\section{SBAs depleted in GP-supplemented mice are FXR agonists that promote expression of ceramide synthesis genes}

The majority of secreted BAs $(>95 \%)$ are reabsorbed in ileum and returned to the liver via the portal vein. ${ }^{7}$ Cecum, colon, and feces have similar BA profiles while the serum BA profile is most closely related to that of ileum and portal vein as a minor fraction of reabsorbed BAs enter systemic circulation. ${ }^{25}{ }^{42}$ We sought to investigate how individual BAs altered by GP supplementation might affect ileal FXR signaling. Gut organoids were cultured from ileal crypts isolated from WT mice (figure 5A) and treated with individual PBAs or SBAs that were differentially detected in serum of GP-supplemented mice (figure 5B). To validate the system, organoids were treated with CDCA, a potent FXR agonist, or CDCA in combination with the FXR antagonist T $\beta$ MCA. As expected, CDCA increased the expression of Fxr, Fgf15, and Shp compared with vehicle-treated organoids and this effect was attenuated by addition of T $\beta M C A$ (figure 5B). When organoids were treated with individual BA alone (ie, $100 \mu \mathrm{M}$ in the absence of CDCA activator), FXR activity was unaffected as $F x$, $F g f 15$, and $S h p$ mRNA expression remained similar to untreated organoids (online supplementary figure 8). We therefore investigated individual BAs in the presence of FXR activator, CDCA, as previously reported, ${ }^{11}$ to reveal agonistic or antagonistic effects on FXR signaling. Organoid cultures treated with CDCA in combination with $\omega \mathrm{MCA}$, TWMCA, THDCA, or DCA increased CDCA-induced expression of Fxr, Fgf15, and $S h p$, indicating that these SBAs are FXR agonists (figure 5B). In agreement with FXR agonistic activity, organoids treated with CDCA in combination with TWMCA resulted in increased expression of $S m p d 3$, Cers 4, and, Sptlc2 ceramide synthesis genes. Cotreatment with CDCA and WMCA, THDCA, or DCA only upregulated Sptlc2 (figure 5B). CA, a PBA reported to be a weak FXR agonist and detected at higher concentration in GP-supplemented $d b / d b$ (but not WT mice), increased CDCA-induced activation of Fxr, $F g f 15$ and Shp and increased expression of Cers 4 and Sptlc2. ${ }^{43}$ TCA, a PBA detected at higher concentration in GP-supplemented $d b / d b$ and WT mice, attenuated CDCA-induced gene expression of $F x r$ and $F g f 15$ but not $S h p$ and reduced CDCA-induced gene expression of Cers 4 (figure 5B). These organoid data suggest that GPs led to the depletion of FXR activators ( $\omega$ MCA, TWMCA and THDCA) and increase of an FXR antagonist (TCA). Consistent with in vivo data, the net effect 
A.

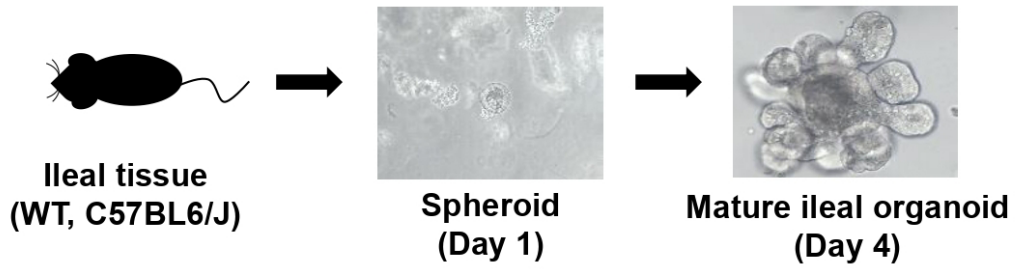

B.

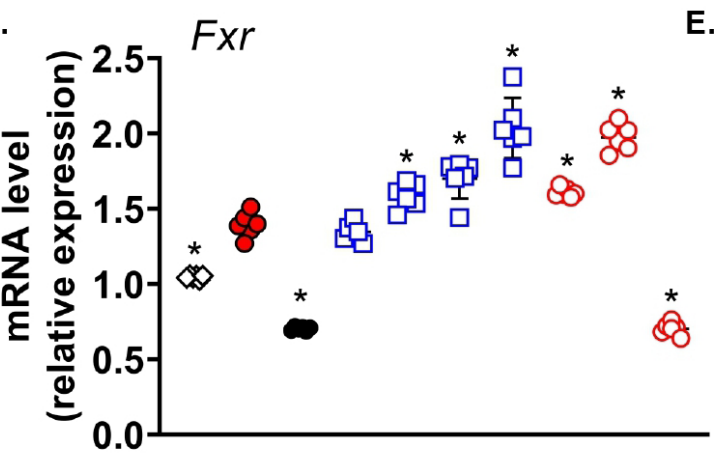

E. Smpd3

C.
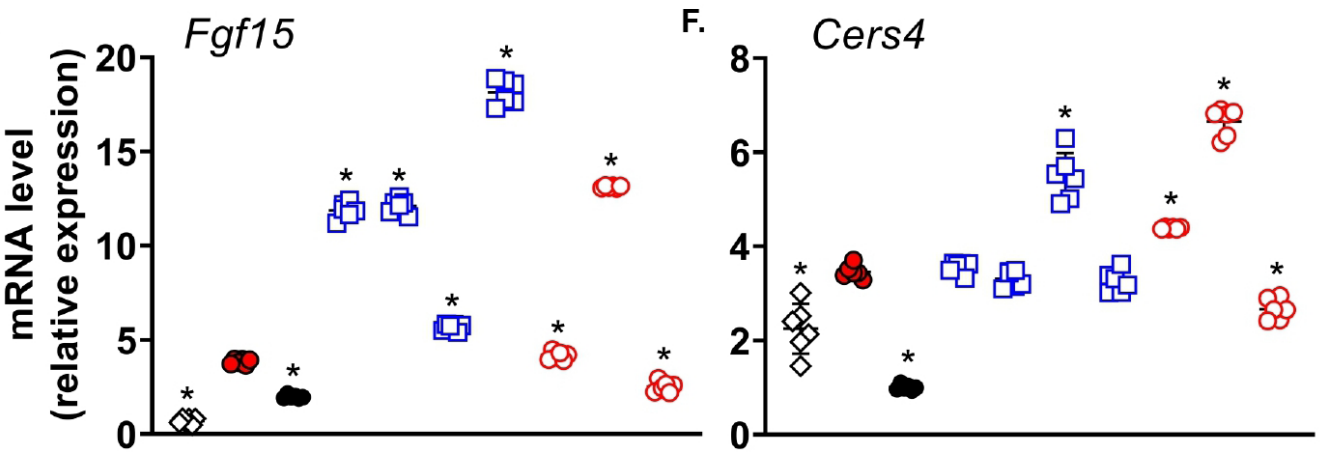

D.
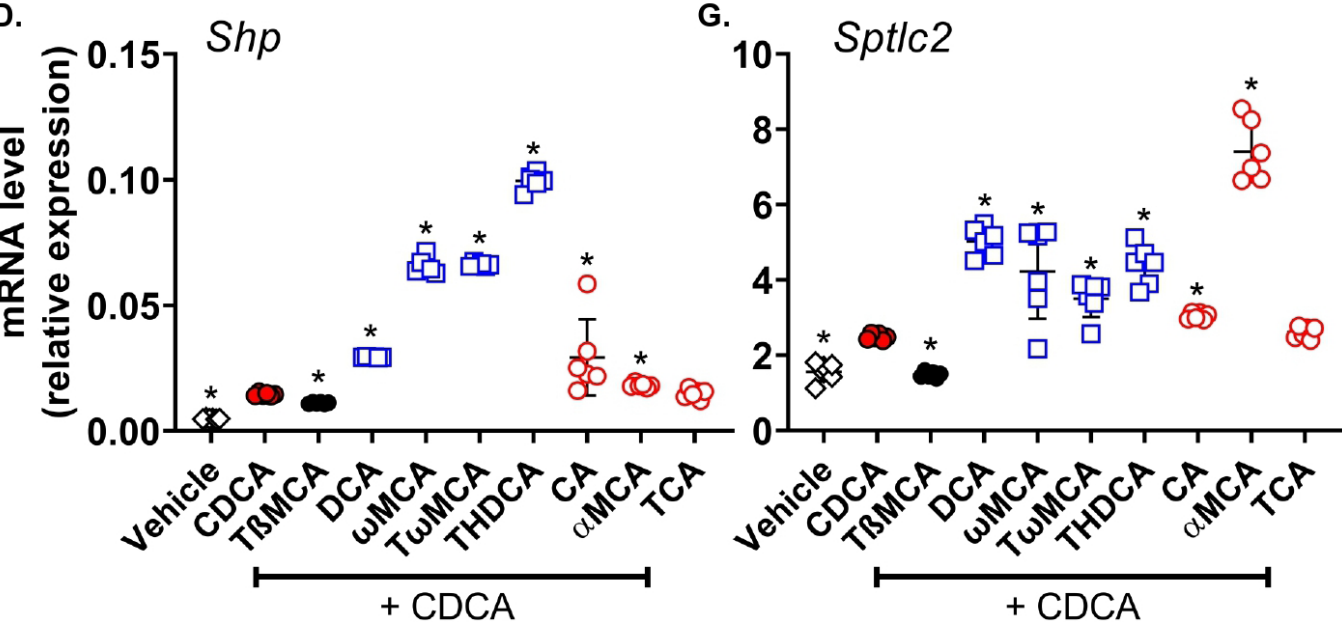

Figure 5 lleal organoids treated with BAs revealed agonistic or antagonistic effects on FXR and ceramide pathway genes (A) lleal crypts were isolated and cultured in Matrigel medium (detailed in methods). Spheroids (representative day 1 photo) matured into organoids (representative day 4 photo). (B) Scatter plot of relative mRNA levels of indicated genes expressed in organoids after 17 hours of treatment with: vehicle (5\% methanol, open black diamonds); $100 \mu \mathrm{M}$ CDCA (closed red circles); a combination of $100 \mu \mathrm{M}$ CDCA and known FXR inhibitor TßMCA $(200 \mu \mathrm{M})$; a combination of $100 \mu \mathrm{M}$ CDCA and indicated PBA $(200 \mu \mathrm{M}$, open red circles), a combination of $100 \mu \mathrm{M}$ CDCA and indicated SBA (200 $\mu \mathrm{M}$, open blue squares). Data shown were combined from two independent experiments, and for each experiment three wells containing mature organoids were treated with indicated BAs. Data represent qPCR of technical duplicates analyzed by $2^{-\Delta C T}$ method. Group mean $\pm S D(n=6$ wells total per treatment group) is illustrated by horizontal and vertical lines. Difference compared with CDCA (FXR agonist) was determined by one-way ANOVA followed by Dunnett's test: ${ }^{*} p<0.05,{ }^{* \star} p<0.01,{ }^{* * *} p<0.001$. ANOVA, analysis of variance; BA, bile acid; CA, cholic acid; CDCA, chenodeoxycholic acid; DCA, deoxycholic acid; FXR, farnesoid X receptor; MCA, muricholic

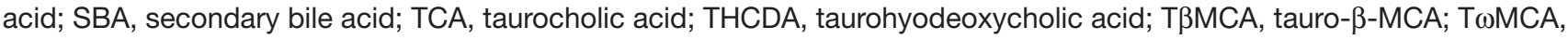
tauro- $\omega-\mathrm{MCA}$; WT, wild type. 
was a BA profile that suppressed FXR signaling and de novo ceramide synthesis.

\section{DISCUSSION}

These data suggest PAC-rich GPs alter a gut microbiotaBA-FXR signaling axis, providing a novel mechanism for glucoregulation. The $d b / d b$ mouse model is characterized by morbid obesity, gut dysbiosis, intestinal barrier dysfunction, gut inflammation, and hyperglycemia allowing investigation of whether a GP-induced bloom in A. muciniphila could ameliorate these metabolic dysfunctions. ${ }^{174}$ We showed that GP-induced improvement in glucose metabolism in $d b / d b$ mice was unrelated to an A. muciniphila-driven reduction of metabolic endotoxemia. These data are consistent with our previous findings in HFD-fed mice supplemented with PAC-rich GPs for 2 weeks where the A. muciniphila bloom and improved glucose metabolism co-occurred, but there were limited positive changes in intestinal gene expression markers associated with metabolic endotoxemia and no significant difference in serum LPS. ${ }^{4}$ Similarly, oral administration of the antidiabetic drug metformin increased A. muciniphila abundance but metabolic benefits were driven by other bacterial species. ${ }^{11456}$ Increased A. muciniphila has been associated with improvements in markers of metabolic endotoxemia. ${ }^{1220}$ The absence of this association in the present study may be due to differences in mouse genotypes (WT, $o b / o b, d b / d b)$ combined with differences in treatment type (probiotic versus prebiotic) and/or duration of dietary supplementation.

In murine models of HFD-induced obesity and accelerated aging, oral administration of $A$. muciniphila correlated with increased intestinal mucus thickness suggesting improved gut barrier integrity. ${ }^{2047}$ In the latter study, gene expression of Muc2 and Muc3 were unchanged while Muc1 was downregulated. ${ }^{47}$ Rats supplemented with wild blueberry PACs showed increased colonic mucus thickness but no difference in mucin gene expression, which is consistent with mucins being regulated mainly at the post-translational level. ${ }^{49}$ In agreement, GP supplementation has consistently shown no increase in $M u c 2$ or $M u c 3$ gene expression; however, mucus thickness was not directly investigated in the present study. ${ }^{14}$ A. muciniphila has generally been associated with decreased gut inflammation and improved health outcomes. However, increasing A. muciniphila abundance is not desirable in all contexts as in other studies it was correlated to mucus depletion, inflammation, and increased susceptibility to enteric pathogen colonization..$^{50-52}$

Concentration of total quantified serum BAs did not differ between groups, but GP-treated $d b / d b$ mice had increased PBAs (CA and TCA) in serum and decreased SBAs (THDCA, WMCA and TWMCA). Administration of the CA was reported to improve energy metabolism in diet-induced obese (DIO) mice. ${ }^{53}$ Increased levels of circulating PBAs correlated with A. muciniphila in association with improved glucose and lipid metabolism in the bile diversion mouse model and improved endothelial function in $\mathrm{Apoe}^{-/-}$mice fed inulin-type fructans. ${ }^{25} 54$ Although our results indicated a positive association between serum PBA and Akkermansia abundance, studies investigating cause-effect relationships are needed. BAs modify the gut microbiota, which in turn dictates BA diversity. The gut microbial alterations we observed likely resulted from combined actions of GPs and BAs. GPs may also influence the gut microbiota and FXR activity by sequestration of BAs, as this activity was recently reported for PACs extracted from persimmon fruit. ${ }^{55}$

GP-treated mice had higher hepatic Cyp7a1 expression compared with controls suggesting increased PBA synthesis contributed to elevated serum $\mathrm{CA}$ and TCA rather than increased ileal BA absorption. Hepatic expression of Cyp7a1 is negatively controlled by intestinal and hepatic FXR activation. ${ }^{28}$ Gene expression of hepatic BA transporters (Abcb11 and Slc51b) and acute phase protein Lcn13 (Obp2a) was similar between groups suggesting GPs did not alter hepatic FXR activity. Intestinal FXR activity was decreased as evidenced by reduced expression of $F g f 15, \operatorname{Sh} p$, and $\operatorname{Tgr} 5$ (figure 4A). Increased Cyp7a1 expression may be mediated by inhibition of intestinal FXR, likely driven by GP-induced changes in gut microbiota and BA diversity. Mice overexpressing Cyp7a1 were protected from DIO, glucose intolerance, insulin resistance and dyslipidemia in association with reduced adiposity and increased energy expenditure via activation of uncoupling protein-1 (UCP-1) in brown adipose tissue. ${ }^{5356}$

Inhibition of ileal FXR represents a key pathway connecting the gut microbiota and BAs with glucose metabolism. Metformin reduced abundance of Bacteroides fragilis in subjects with T2D resulting in increased circulating levels of glycoursodeoxycholic acid, which inhibited intestinal FXR leading to improved serum glucose levels. ${ }^{11}$ Previous studies in DIO mice showed that inhibition of intestinal FXR by caffeic acid phenethyl ester, tempol, or antibiotics improved glycemia by decreasing synthesis of intestinal ceramides and their subsequent migration via lymph to the liver where they promote oxidative stress, insulin resistance, and gluconeogenesis. ${ }^{10}{ }^{36}$ GP-supplementation resulted in downregulation of Smpd3 in the intestine and Sptlc2 and Cers 4 in liver concomitant with diminished mRNA levels of genes involved in hepatic lipogenesis (Chrebp), gluconeogenesis (G6pase), $\beta$-oxidation (Idhl3a), and inflammation ( $L b p)$. A recent study using loss and gain of function models showed that decreasing hepatic ceramides improved glucose tolerance, insulin resistance, and reduced hepatic steatosis in mice. ${ }^{37}$ As GP-treated mice had lower expression of ceramide synthesis genes, improvements in glucose metabolism may be linked to lower hepatic ceramide levels; however, studies using intestine-specific FXR $\mathrm{KO}$ mice and gnotobiotic/antibiotic-treated mice and ceramide quantification are needed for confirmation. 
Different types or mixtures of dietary polyphenols likely have differential effects on the gut microbiota, BA profile, and FXR signaling. ${ }^{57}$ In Caco-2 cells, PACs inhibited FXR signaling and p-coumaric acid, a microbial-derived PAC metabolite, inhibited FXR in a yeast 2-hybrid assay. ${ }^{58} 59$ PACs were reported to directly modulate FXR signaling and attenuate hypertriglyceridemia but it remains unclear whether this effect was due to FXR activation or suppression. ${ }^{5860}$ FXR signaling has been reported to both induce and prevent metabolic impairments, underlining its complex role in host energy metabolism. ${ }^{28}$ Organoid experiments suggested that SBAs THDCA, WMCA, T $\omega \mathrm{MCA}$ and PBA $\alpha \mathrm{MCA}$ are FXR agonists (figure 5). GP-induced depletion of these agonistic BAs in vivo is therefore consistent with the observed downregulation of Fxr-responsive genes ( $F g f 15, S h p, T g r 5)$ in ileum tissue. Moreover, GP supplementation increased levels of serum TCA, an FXR antagonist in our organoid system. TCA was also reported as an FXR agonist $;{ }^{2}$ however, this discrepancy does not appear to be due to our experimental conditions as the activities of well-established FXR agonist CDCA and antagonist T $\beta M C A$ were highly reproducible in our system. GP supplementation induced a state of FXR inhibition despite increased concentrations of DCA and CA, which was reported to be a weak FXR agonist. ${ }^{43}$ These data suggest that GP-induced FXR inhibition is determined by the overall balance of agonistic and inhibitory BAs. While our data suggest that GP-induced depletion of agonistic SBAs resulted in FXR inhibition, the exact mechanism(s) involved warrant further investigation.

In summary, PAC-rich GPs reduced gut bacteria associated with production of SBAs identified as FXR agonists leading to FXR inhibition and improved glucose metabolism, potentially via reduced ceramide biosynthesis. These findings suggest a BA-FXR signaling axis as a putative novel mechanism to explain glucoregulatory effects of dietary PACs.

Acknowledgements We thank LZ for assistance with heatmap code, Henrik Munch Roager for BA analysis consultations, Shikha Ranka and Madeline Bandomer for technical support.

Contributors KMT, JAVR, and DER planned and designed the experiments. DER performed murine experiments. KMT performed targeted LC-MS and prepared 16S amplicon libraries. KMT and LZ analyzed amplicon data. KMT, AJC, FGB, and JAVR performed qPCR. JAVR and AJC performed organoid experiments. RMD provided technical support. KMT and JAVR drafted the paper. DER oversaw project and edited final manuscript. All authors read and approved the final manuscript. DER is the guarantor of this work and, as such, had full access to all the data in the study and takes responsibility for the integrity of the data and the accuracy of the data analysis.

Funding This work was supported by NIH-NCCIH grants R01 AT010242, K01 AT008829, and 2P50AT002776-11S03 to DER.

Competing interests DER has equity in Nutrasorb LLC.

Patient consent for publication Not required.

Provenance and peer review Not commissioned; externally peer reviewed.

Data availability statement Data are available on reasonable request. All data relevant to the study are included in the article or uploaded as supplementary information. DNA sequences encoding bacterial and archaeal 16S rRNA V4 region reported in this paper have been deposited in the Sequence Read Archive under the accession number PRJNA622906.

Open access This is an open access article distributed in accordance with the Creative Commons Attribution Non Commercial (CC BY-NC 4.0) license, which permits others to distribute, remix, adapt, build upon this work non-commercially, and license their derivative works on different terms, provided the original work is properly cited, appropriate credit is given, any changes made indicated, and the use is non-commercial. See: http://creativecommons.org/licenses/by-nc/4.0/.

\section{ORCID iDs}

Jose A Villa-Rodriguez http://orcid.org/0000-0002-7189-5505

Diana E Roopchand http://orcid.org/0000-0002-4165-2377

\section{REFERENCES}

1 Roopchand DE, Carmody RN, Kuhn P, et al. Dietary Polyphenols Promote Growth of the Gut Bacterium Akkermansia muciniphila and Attenuate High-Fat Diet-Induced Metabolic Syndrome. Diabetes 2015;64:2847-58.

2 Anhê FF, Roy D, Pilon G, et al. A polyphenol-rich cranberry extract protects from diet-induced obesity, insulin resistance and intestinal inflammation in association with increased Akkermansia spp. population in the gut microbiota of mice. Gut 2015;64:872-83.

3 Rienks J, Barbaresko J, Oluwagbemigun K, et al. Polyphenol exposure and risk of type 2 diabetes: dose-response meta-analyses and systematic review of prospective cohort studies. Am J Clin Nutr 2018;108:49-61.

4 Zhang L, Carmody RN, Kalariya HM, et al. Grape proanthocyanidininduced intestinal Bloom of Akkermansia muciniphila is dependent on its baseline abundance and precedes activation of host genes related to metabolic health. J Nutr Biochem 2018;56:142-51.

5 Depommier C, Everard A, Druart C, et al. Supplementation with Akkermansia muciniphila in overweight and obese human volunteers: a proof-of-concept exploratory study. Nat Med 2019;25:1096-103.

6 Plovier H, Everard A, Druart C, et al. A purified membrane protein from Akkermansia muciniphila or the pasteurized bacterium improves metabolism in obese and diabetic mice. Nat Med 2017;23:107-13.

7 Chiang JYL, Ferrell JM. Bile Acids as Metabolic Regulators and Nutrient Sensors. In: Annual review of nutrition. , 2019: 39, 175-200.

8 Parséus A, Sommer N, Sommer F, et al. Microbiota-Induced obesity requires farnesoid X receptor. Gut 2017;66:429-37.

9 Li F, Jiang C, Krausz KW, et al. Microbiome remodelling leads to inhibition of intestinal farnesoid $X$ receptor signalling and decreased obesity. Nat Commun 2013;4:2384.

10 Jiang C, Xie C, Li F, et al. Intestinal farnesoid X receptor signaling promotes nonalcoholic fatty liver disease. J Clin Invest 2015;125:386-402.

11 Sun L, Xie C, Wang G, et al. Gut microbiota and intestinal FXR mediate the clinical benefits of metformin. Nat Med 2018;24:1919-29.

12 Jiang C, Xie C, Lv Y, et al. Intestine-selective farnesoid X receptor inhibition improves obesity-related metabolic dysfunction. Nat Commun 2015;6:10166.

13 Roopchand DE, Kuhn P, Krueger CG, et al. Concord grape pomace polyphenols complexed to soy protein isolate are stable and hypoglycemic in diabetic mice. J Agric Food Chem 2013;61:11428-33.

14 Callahan BJ, McMurdie PJ, Rosen MJ, et al. DADA2: high-resolution sample inference from illumina amplicon data. Nat Methods 2016;13:581-3.

15 Bolyen E, Rideout JR, Dillon MR, et al. Reproducible, interactive, scalable and extensible microbiome data science using QIIME 2. Nat Biotechnol 2019;37:852-7.

16 Mahe MM, Aihara E, Schumacher MA, et al. Establishment of gastrointestinal epithelial organoids. Curr Protoc Mouse Biol 2013;3:217-40.

17 Thaiss CA, Levy M, Grosheva I, et al. Hyperglycemia drives intestinal barrier dysfunction and risk for enteric infection. Science 2018;359:1376-83.

18 Kennedy EA, King KY, Baldridge MT. Mouse microbiota models: comparing germ-free mice and antibiotics treatment as tools for modifying gut bacteria. Front Physiol 2018;9:1534.

19 Mayer R, Stecher G, Wuerzner R, et al. Proanthocyanidins: target compounds as antibacterial agents. J Agric Food Chem 2008;56:6959-66.

20 Everard A, Belzer C, Geurts L, et al. Cross-Talk between Akkermansia muciniphila and intestinal epithelium controls dietinduced obesity. Proc Natl Acad Sci U S A 2013;110:9066-71. 
21 Kakiyama G, Pandak WM, Gillevet PM, et al. Modulation of the fecal bile acid profile by gut microbiota in cirrhosis. $J$ Hepatol 2013;58:949-55

22 Wells JE, Williams KB, Whitehead TR, et al. Development and application of a polymerase chain reaction assay for the detection and enumeration of bile acid 7alpha-dehydroxylating bacteria in human feces. Clin Chim Acta 2003;331:127-34.

23 Ridlon JM, Kang DJ, Hylemon PB, et al. Bile acids and the gut microbiome. Curr Opin Gastroenterol 2014;30:332-8.

24 Ridlon JM, Kang D-J, Hylemon PB. Bile salt biotransformations by human intestinal bacteria. J Lipid Res 2006;47:241-59.

25 Catry E, Bindels LB, Tailleux A, et al. Targeting the gut microbiota with inulin-type fructans: preclinical demonstration of a novel approach in the management of endothelial dysfunction. Gut 2018;67:271-83.

26 Kim Y-C, Fang S, Byun S, et al. Farnesoid X receptor-induced lysine-specific histone demethylase reduces hepatic bile acid levels and protects the liver against bile acid toxicity. Hepatology 2015;62:220-31.

27 Inagaki T, Choi M, Moschetta A, et al. Fibroblast growth factor 15 functions as an enterohepatic signal to regulate bile acid homeostasis. Cell Metab 2005;2:217-25.

28 Shapiro $\mathrm{H}$, Kolodziejczyk AA, Halstuch $\mathrm{D}$, et al. Bile acids in glucose metabolism in health and disease. J Exp Med 2018;215:383-96.

29 Kim Y-C, Byun S, Zhang Y, et al. Liver ChIP-Seq analysis in FGF19treated mice reveals SHP as a global transcriptional partner of SREBP-2. Genome Biol 2015;16:268.

30 Sticova E, Jirsa M, Pawłowska J. New insights in genetic cholestasis: from molecular mechanisms to clinical implications. Can $J$ Gastroenterol Hepatol 2018;2018:1-12.

31 Eloranta JJ, Kullak-Ublick GA. The role of FXR in disorders of bile acid homeostasis. Physiology 2008;23:286-95.

32 Armstrong LE, Guo GL. Role of FXR in liver inflammation during nonalcoholic steatohepatitis. Curr Pharmacol Rep 2017:3:92-100.

33 Pathak $\mathrm{P}$, Liu $\mathrm{H}$, Boehme $\mathrm{S}$, et al. Farnesoid $\mathrm{X}$ receptor induces Takeda G-protein receptor 5 cross-talk to regulate bile acid synthesis and hepatic metabolism. J Biol Chem 2017;292:11055-69.

34 Paternoster S, Falasca M. Dissecting the physiology and pathophysiology of glucagon-like peptide-1. Front Endocrinol 2018;9:584.

35 Chaurasia B, Summers SA. Ceramides - Lipotoxic Inducers of Metabolic Disorders. Trends Endocrinol Metab 2015;26:538-50.

36 Xie C, Jiang C, Shi J, et al. An intestinal farnesoid X ReceptorCeramide signaling axis modulates hepatic gluconeogenesis in mice. Diabetes 2017;66:613-26.

37 Chaurasia B, Tippetts TS, Mayoral Monibas R, et al. Targeting a ceramide double bond improves insulin resistance and hepatic steatosis. Science 2019;365:386-92.

38 Ortega-Prieto P, Postic C. Carbohydrate sensing through the transcription factor ChREBP. Front Genet 2019;10:472.

39 Tommasini-Ghelfi S, Murnan K, Kouri FM, et al. Cancer-Associated mutation and beyond: the emerging biology of isocitrate dehydrogenases in human disease. Sci Adv 2019;5:eaaw4543.

40 Moreno-Navarrete JM, Ortega F, Serino M, et al. Circulating lipopolysaccharide-binding protein (LBP) as a marker of obesityrelated insulin resistance. Int J Obes 2012;36:1442-9.

41 Chikka MR, McCabe DD, Tyra HM, et al. C/Ebp homologous protein (CHOP) contributes to suppression of metabolic genes during endoplasmic reticulum stress in the liver. $\mathrm{J}$ Biol Chem 2013;288:4405-15.

42 Sayin SI, Wahlström A, Felin J, et al. Gut microbiota regulates bile acid metabolism by reducing the levels of tauro-beta- muricholic acid, a naturally occurring FXR antagonist. Cell Metab 2013:17:225-35

43 Makishima M, Okamoto AY, Repa JJ, et al. Identification of a nuclear receptor for bile acids. Science 1999;284:1362-5

44 Rajala MW, Patterson CM, Opp JS, et al. Leptin acts independently of food intake to modulate gut microbial composition in male mice. Endocrinology 2014;155:748-57.

45 de la Cuesta-Zuluaga J, Mueller NT, Corrales-Agudelo V, et al. Metformin is associated with higher relative abundance of mucin-degrading Akkermansia muciniphila and several shortchain fatty acid-producing microbiota in the gut. Diabetes Care 2017:40:54-62.

46 Shin $\mathrm{N}-\mathrm{R}$, Lee $\mathrm{J}-\mathrm{C}$, Lee $\mathrm{H}-\mathrm{Y}$, et al. An increase in the Akkermansia spp. population induced by metformin treatment improves glucose homeostasis in diet-induced obese mice. Gut 2014;63:727-35.

47 van der Lugt B, van Beek AA, Aalvink S, et al. Akkermansia muciniphila ameliorates the age-related decline in colonic mucus thickness and attenuates immune activation in accelerated aging Ercc $^{-/ \Delta 7}$ mice. Immun Ageing 2019;16:6.

48 Rodríguez-Daza M-C, Daoust L, Boutkrabt L, et al. Wild blueberry proanthocyanidins shape distinct gut microbiota profile and influence glucose homeostasis and intestinal phenotypes in high-fat high-sucrose fed mice. Sci Rep 2020;10:2217.

49 Schroeder BO. Fight them or feed them: how the intestinal mucus layer manages the gut microbiota. Gastroenterol Rep 2019;7:3-12.

50 Zella GC, Hait EJ, Glavan T, et al. Distinct microbiome in pouchitis compared to healthy pouches in ulcerative colitis and familial adenomatous polyposis. Inflamm Bowel Dis 2011;17:1092-100.

51 Ganesh BP, Klopfleisch R, Loh G, et al. Commensal Akkermansia muciniphila exacerbates gut inflammation in Salmonella typhimurium-infected gnotobiotic mice. PLoS One 2013;8:e74963.

52 Weir TL, Manter DK, Sheflin AM, et al. Stool microbiome and metabolome differences between colorectal cancer patients and healthy adults. PLoS One 2013;8:e70803.

53 Watanabe M, Houten SM, Mataki C, et al. Bile acids induce energy expenditure by promoting intracellular thyroid hormone activation. Nature 2006;439:484-9.

54 Pierre JF, Martinez KB, Ye H, et al. Activation of bile acid signaling improves metabolic phenotypes in high-fat diet-induced obese mice. Am J Physiol Gastrointest Liver Physiol 2016;311:G286-304.

55 Hamauzu Y, Suwannachot J. Non-Extractable polyphenols and in vitro bile acid-binding capacity of dried persimmon (Diospyros kaki) fruit. Food Chem 2019;293:127-33.

$56 \mathrm{Li} \mathrm{T}$, Owsley E, Matozel M, et al. Transgenic expression of cholestero 7alpha-hydroxylase in the liver prevents high-fat diet-induced obesity and insulin resistance in mice. Hepatology 2010;52:678-90.

57 Villa-Rodriguez JA, Ifie I, Gonzalez-Aguilar GA, et al. The gastrointestinal tract as prime site for cardiometabolic protection by dietary polyphenols. Adv Nutr 2019;10:999-1011.

58 Heidker RM, Caiozzi GC, Ricketts M-L. Dietary procyanidins selectively modulate intestinal farnesoid $X$ receptor-regulated gene expression to alter enterohepatic bile acid recirculation: elucidation of a novel mechanism to reduce triglyceridemia. Mol Nutr Food Res 2016;60:727-36.

59 Diao Y, Jiang J, Zhang S, et al. Discovery of natural products as novel and potent FXR antagonists by virtual screening. Front Chem 2018;6:140.

60 Del Bas JM, Ricketts M-L, Vaqué M, et al. Dietary procyanidins enhance transcriptional activity of bile acid-activated FXR in vitro and reduce triglyceridemia in vivo in a FXR-dependent manner. Mol Nutr Food Res 2009;53:805-14. 